\title{
Novel anti-aging composition for topical skin care
}

\author{
Dariusz WaWrzyniaK ${ }^{1}$, Lech CelewiCZ ${ }^{2}$, JAN BARCiSzeWSKi ${ }^{1 *}$ \\ ${ }^{1}$ Institute of Bioorganic Chemistry, Polish Academy of Sciences, Poznań, Poland \\ ${ }^{2}$ Faculty of Chemistry, Adam Mickiewicz University in Poznan, Poznań, Poland
}

US Patent

Patent No.: US 7923578 B2

Title of invention: Method of manufacturing 3-(4-hydroxyphenyl)propanoic acid amide, its application in the manufacture of anti-aging compositions and anti-aging composition

Inventors: J. Barciszewski, L. Rychlewski, L. Celewicz, K. Ciszewski, E. Wyszko, K. Rolle

Assignee: Institute of Bioorganic Chemistry, PAS, Poznań

Date of patent: 12.04 .2011

European patent

PCT number: PCT/PL2006/000069

Title of invention: Method of manufacturing 3-(4-hydroxyphenyl)propanoic acid amide, its application in the manufacture of anti-aging compositions and anti-aging composition

Inventors: J. Barciszewski, L. Rychlewski, L. Celewicz, K. Ciszewski, E. Wyszko, K. Rolle

Assignee: Institute of Bioorganic Chemistry, PAS, Poznań

Application date: 18.10 .2006

Published also as: EP1971571A2, EP1971571B1, US20080249334, WO2007046721A2, WO2007046721A3

\begin{abstract}
The subject of the invention is a method of manufacturing 3-(4-hydroxyphenyl)propanoic acid amide and its application in the preparation of anti-aging compositions. Due to the biological properties of phloretamide, it is used in the preparation of skin care composition that has excellent anti-aging effect in preventing skin wrinkles.
\end{abstract}

Key words: anti-aging composition, topical skin care, 3-(4-hydroxyphenyl)propanoic acid amide

\section{State of the art}

One of the most common dermatologic concerns among patients is skin aging. In a culture that is often called "youth-obsessed", individuals increasingly look for creams and procedures that can improve the appearance of their skin. According to Mintel (a market research company), the US anti-aging skin care market generated about 2.11 billion U.S. dollars in 2013 (Choi and Berson 2006).

Studies have extensively focused on the appearance of unpleasant, observable marks on the skin's surface; this is due to the proteolysis of cutaneous elastic fibers, which results in reduced cell functions. Skin aging can be divided into two types based on the physiological and environmental factors, respectively-intrinsic aging or chronological aging (inevitable phenomenon) and extrinsic or premature or photoaging (evitable phenomenon) (Braverman and Fonferko 1982; Montagna et al. 1989; Warren et al. 1991). Clinical signs of photoaging include dryness, irregular and dark/light pigmentation, sallow skin, either deep furrows or severe atrophy, telangiectasias, premalignant lesions, laxity, and a leathery appearance (Yaar et al. 2002). The primary cause of skin damage is oxidative stress caused by UVA light. UVA is absorbed by chromophores present in the skin (e.g. trans-urocanic acid), which generate various reactive oxygen species (ROS) that cause oxidative damage to nucleic acids, cellular proteins, and lipids (Hanson and 
Simon 1998). The ROS triggers a host of cytokine cascades that result in photoaging and photocarcinogenesis. One of the primary effects of UVA oxidative damage is ROS-induced synthesis of a series of matrix metalloproteinases that cause collagen degradation resulting in wrinkles (Fisher et al. 2001). Chronologic skin aging, in contrast, is characterized by laxity and fine wrinkling, as well as development of benign growth such as seborrheic keratoses and angiomas, but it is not associated with increased pigmentation or deep wrinkles that characterize photoaging (Yaar et al. 2002). It is also important to note that the genetic program of intrinsic aging differs per individual in terms of rate and severity of effect.

Skin care, and particularly anti-aging strategies, is a substantial and growing industry that encompasses a range of disciplines such as cosmetic, pharmaceutical, and physical therapy. As the population ages, they wish to maintain a youthful vigor and appearance; employing anti-aging strategies is an option for an individual to retain youthful appearance. A large portion of this antiaging strategy is skin treatment, the skin being the most visible indicia of aging. As a result, consumer constantly focus on the newest compositions and methods to defy the effects of skin aging, counteract wrinkles, acquire a smooth and bright skin, and obtain additional antiaging benefits (Ganceviciene et al. 2012).

Surgery is one strategy to achieve a relatively fast improvement in the skin's appearance. However, surgery is expensive, dangerous, and has obvious drawbacks. A more popular approach is to apply a cosmetic formulation to the skin to counter the effects of aging (Baumann 2007). Treatments designed to prolong or promote youthful appearance include topical applications of cosmetic preparations, lotions and moisturizers, electrical stimulation, and collagen injections (Tabata et al. 2000). Several cosmetic ingredients, such as Botox ${ }^{\circledR}$ (botulinum toxin), have been used to improve the appearance of the skin via their skin-beneficiary effects that include skin resurfacing, skin wrinkle reduction, and anti-aging effects. Such cosmetic ingredients typically are applied directly to the skin. However, these topical formulations require a consistent and extended regimen before results are observed. These formulations also can be invasive and generate side effects (Dessy et al. 2007; Rzany et al. 2007).

Small molecules are one of the novel agents that are most promising and hence could be subjected to use in cosmetology and dermatology. Kinetin $\left(\mathrm{N}^{6}\right.$-furfurylade- nine) is a cosmeceutical agent that is used in the prevention and treatment of skin aging. It is a naturally occurring plant growth factor that retards senescence in plants and has been found to have anti-aging effects on human adult skin fibroblasts in vitro (Barciszewski et al. 1999; Rattan and Clark 1994). Kinetin appears to be both a direct anti-oxidant and a signaling molecule that stimulates the maintenance and repair pathways in cells (Olsen et al. 1999; Verbeke et al. 2000). Extended treatment with kinetin was well tolerated and did not cause irritation. Other small molecules obtained from plants may also be useful in the prevention, intervention, and therapy of aging (Rattan and Sodagam 2005).

Phenolic compounds are secondary plant metabolites produced in response to various stresses such as infections, wounding, UV irradiation, and exposure to ozone and pollutants. They are the most abundant and biologically active phytonutrients, which reduce inflammation, inhibit tumor growth, have pro-apoptotic and anti-angiogenic properties, possess anti-microbial, antiviral, and anti-aging properties, modulate immune system, increase capillary resistance, protect cardiovascular and neurological systems, limit weight gain, promote wound healing, and so on (Munin and EdwardsLevy 2011). One of these compounds, 3-(4-hydroxyphenyl)propanoic acid amide, possesses a wide range of biological properties. Low-molecular-mass compounds in plant juices play a very significant role in the growth and development of plant shoots. The pulp of Malus domestica (cv. Lobo) was shown to contain 3-(4-hydroxyphenyl)propanoic acid amide, also called phloretamide (No. CA 23838-70-2), identified by mass spectrometry and NMR spectroscopy (Rybicka 1984). The important point to note is that secreted plant juice has a much higher concentration of phloretamide than the internal plant juices (Rybicka 1996). Structurally similar compounds, such as hydroxycinnaminic acids, are some of the major phenolic compounds that occur in plants. In particular, $p$-hydroxycinnamic acid is a common component that occurs in higher plants (Bearder 1980). Hydroxycinnamic acids were determined to possess diverse biological properties such as the following: they possess antioxidant, anti-apoptosis, anti-aging, anti-carcinogen, anti-inflammation, and anti-atherosclerosis properties; provide cardiovascular protection; improve endothelial function, and inhibit angiogenesis and cell proliferation activity (Han et al. 2007). 
<smiles>O=C([OH2+])CCc1ccc(O)cc1</smiles><smiles>CCOC(C)=O</smiles><smiles>O=C(CCc1ccc(O)cc1)Oc1ccc([N+](=O)[O-])cc1</smiles>

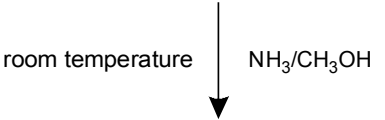<smiles>NC(=O)CCc1ccc(O)cc1</smiles>

Fig. 1. Synthesis of 3-(4-hydroxyphenyl)propanoic acid amide

To date, all the methods that have addressed the problems of improving the appearance and condition of aged skin have their own drawbacks. Therefore, there remains a need in the art for skin-care compositions that are capable of maintaining or restoring a youthful appearance in aging skin and/or improving the condition and appearance of aging skin. The goal of the present invention was to develop a method to manufacture 3-(4hydroxyphenyl)propanoic acid amide and its further application in the cosmetic and pharmaceutical industries in the preparation of an anti-aging composition, which has an excellent anti-aging effect that prevents the sagging of skin and loss of luster.

\section{The patent's claims}

- A method of manufacturing 3-(4-hydroxyphenyl)propanoic acid amide characterized that the synthesis of 3-(4-hydroxyphenyl)propanoic acid amide is performed using active 4-nitrophenyl ester where the concentration of 3-(4-hydroxyphenyl)propanoic acid is $0.12-0.0012 \mathrm{~mol}$, where the reaction is performed with ammonia at room temperature, and the 4-nitrophenol in ethyl acetate is supplemented with $\mathrm{N}, \mathrm{N}$ - dicyclohexylocarbodiimide, then the reaction mixture is stirred, the N,N-dicyclohexylurea precipitate is then filtered out and dried under vacuum, the remainder is supplemented with anhydrous methanol saturated with ammonia $\left(10 \mathrm{~cm}^{3}\right)$ and the reaction mixture is stirred and evaporated until dry, then the remainder is loaded onto a silica gel chromatography column, and the latter is washed with chloroformmethanol $(50: 1, \mathrm{v} / \mathrm{v})$, whence the chromatographically clean product is crystallized from methanol and then dried in a vacuum dessicator over phosphorus pentoxideyielding 3-(4-hydroxyphenyl)propanoic acid amide. The method of manufacturing 3-(4-hydroxyphenyl)propanoic acid amide is presented in Figure 1.

- Preferentially, 0.5-5 g of 3-(4-hydroxyphenyl)propanoic acid solution is used, with 0.5-5 g 4-nitrophenol in ethyl oxide, 5-15 times excess N,N-dicyclohexylcarbodiimide in relation to 4-nitrophenol, and the anhydrous methanol saturated with ammonia is between 5 and $30 \mathrm{~cm}^{3}$, wherein the reaction mixture is mixed in a temperature range $0-10^{\circ} \mathrm{C}$ for $10-50 \mathrm{~min}$, whereafter it is mixed at room temperature for 1.5-3 $\mathrm{h}$, then the precipitated $\mathrm{N}, \mathrm{N}$-dicyclohexylurea is filtered out and washed in $2-15 \mathrm{~cm}^{3}$ ethyl acetate.

- Application of 3-(4-hydroxyphenyl)propanoic acid amide in the manufacture of anti-aging compositions.

- A cosmetic or pharmaceutical composition comprising 3-(4-hydroxyphenyl)propanoic acid amide at a concentration between 1 and $500 \mu \mathrm{mol}$.

\section{Conclusions}

A series of experiments carried out on the shortterm effects of phloretamide on human skin fibroblasts indicate that this compound has significant beneficial anti-aging effects. At concentrations of 40 and $80 \mu \mathrm{M}$, enhanced cell attachment (10-20\%) along with stimulation of cell growth was observed through a one-step growth-curve analysis (up to 11 days).

\section{References}

Barciszewski J., Rattan S.I.S., Siboska G., Clark B.F.C. (1999) Kinetin - 45 years on. Plant Sci. 148(1): 37-45.

Baumann L. (2007) Skin ageing and its treatment. J. Pathol. 211(2): 241-251.

Bearder J.R. (1980) Plant hormones and other growth substances - their background, structures and occurrence. 
[In:] Hormonal regulation of development I. Ed. MacMillan J. Springer, Berlin Heidelberg: 9-112.

Braverman I.M., Fonferko E. (1982) Studies in cutaneous aging: I. The elastic fiber network. J. Invest. Dermatol. 78(5): 434-443.

Choi C.M., Berson D.S. (2006) Cosmeceuticals. Semin. Cutan. Med. Surg. 25(3): 163-168.

Dessy L.A., Mazzocchi M., Rubino C., Mazzarello V., Spissu N., Scuderi N. (2007) An objective assessment of botulinum toxin A effect on superficial skin texture. Ann. Plast. Surg. 58(5): 469-473.

Fisher G.J., Choi H.C., Bata-Csorgo Z., Shao Y., Datta S., Wang Z.Q., Kang S., Voorhees J.J. (2001) Ultraviolet irradiation increases matrix metalloproteinase- 8 protein in human skin in vivo. J. Invest. Dermatol. 117(2): 219-226.

Ganceviciene R., Liakou A.I., Theodoridis A., Makrantonaki E., Zouboulis C.C. (2012) Skin anti-aging strategies. Dermatoendocrinol. 4(3): 308-319.

Han X., Shen T., Lou H. (2007) Dietary polyphenols and their biological significance. Int. J. Mol. Sci. 8(9): 950-988.

Hanson K.M., Simon J.D. (1998) Epidermal trans-urocanic acid and the UV-A-induced photoaging of the skin. Proc. Natl. Acad. Sci. USA 95(18): 10576-10578.

Montagna W., Kirchner S., Carlisle K. (1989) Histology of sundamaged human skin. J. Am. Acad. Dermatol. 21(5 Pt. 1): 907-918.

Munin A., Edwards-Levy F. (2011) Encapsulation of natural polyphenolic compounds; a review. Pharmaceutics 3(4): 793-829.

Olsen A., Siboska G.E., Clark B.F., Rattan S.I. (1999) N(6)Furfuryladenine, kinetin, protects against Fenton reaction-mediated oxidative damage to DNA. Biochem. Biophys. Res. Commun. 265(2): 499-502.
Rattan S.I., Clark B.F. (1994) Kinetin delays the onset of ageing characteristics in human fibroblasts. Biochem. Biophys. Res. Commun. 201(2): 665-672.

Rattan S.I., Sodagam L. (2005) Gerontomodulatory and youthpreserving effects of zeatin on human skin fibroblasts undergoing aging in vitro. Rejuvenation Res. 8(1): 46-57.

Rybicka H. (1984) 3-[p-Hydroxyphenyl]-proprionic acid amide (Phloretamide) in root exudate of malus. Biochem. Physiol. Pflanzen 179: 303-309.

Rybicka H. (1996) Phloretamid in fruitlets of apple tree (Malus domestica). Acta Physiol. Plant. 18(4): 359-363.

Rzany B., Dill-Muller D., Grablowitz D., Heckmann M., Caird D., German-Austrian Retrospective Study G. (2007) Repeated botulinum toxin $A$ injections for the treatment of lines in the upper face: a retrospective study of 4,103 treatments in 945 patients. Dermatol. Surg. 33(1 Spec. No.): S18-25.

Tabata N., O’Goshi K., Zhen Y.X., Kligman A.M., Tagami H. (2000) Biophysical assessment of persistent effects of moisturizers after their daily applications: evaluation of corneotherapy. Dermatology 200(4): 308-313.

Verbeke P., Siboska G.E., Clark B.F., Rattan S.I. (2000) Kinetin inhibits protein oxidation and glycoxidation in vitro. Biochem. Biophys. Res. Commun. 276(3): 1265-1270.

Warren R., Gartstein V., Kligman A.M., Montagna W., Allendorf R.A., Ridder G.M. (1991) Age, sunlight, and facial skin: a histologic and quantitative study. J. Am. Acad. Dermatol. 25(5 Pt. 1): 751-760.

Yaar M., Eller M.S., Gilchrest B.A. (2002) Fifty years of skin aging. J. Investig. Dermatol. Symp. Proc. 7(1): 51-58. 